\title{
An Evaluation of the Relative Distribution of Romanian Patients with Pancreatic Neoplasm TIMOFTE Daniel $^{1 *}$, IONESCU Lidia ${ }^{1}$, OCHIUZ Lacramioara ${ }^{1}$ \\ 1 "Gr. T. Popa” University of Medicine and Pharmacy, lasi, Romania \\ *dantimofte@yahoo.com
}

Keywords: cancer, pancreatic.

\begin{abstract}
This study describes the specific features of the distribution of Romanian patients with pancreatic neoplasm. The analysis was made on 188 patients from Clinical Emergency Hospital "Sf. Spiridon, Iasi", Romania, all with solid form of pancreatic cancer. Moreover, the distribution by age, gender, tumor location in the pancreas or by the stages from the TNM system was analyzed and compared with other studies from other countries. Thus, in our sample the median age was 65 years old. In addition, $53 \%$ of the patients had rural origin and $47 \%$ urban origin. Also, regarding the pancreatic tumor location, by far the most frequent was the cephalic location $(75 \%)$. Moreover, when we study the distribution by presence of metastases at the time of diagnosis, we observed that $62.1 \%$ are located in the liver and $21.2 \%$ into the peritoneum. In addition, when we staged the ductal adenocarcinoma of the pancreas according to the TNM (tumour grade, nodal status and metastases) system, where T1-T3 constitutes a resectable tumour and T4 indicates an unresectable tumour, we found that group T4 stage tumor predominated (63.8\%), while in regards to the regional lymph nodes (e.g. $\mathrm{N}$ in TNM classification) we observed that in an overwhelming proportion $\mathrm{Nx}$ stage predominates $(88.6 \%)$. Moreover, no distant metastasis was found in $63 \%$ of patients, by using $\mathrm{M}$ in TNM classification. In this way, a better understanding of the specific characteristics for the affected population could prove to be extremely important, especially considering the fact during the last decades the development of the surgical methods and techniques for this disorder had no significant impact on the survival rate.
\end{abstract}

\section{Introduction}

Pancreatic ductal adenocarcinoma has the worst prognosis of all forms of gastrointestinal cancers [1]. The incidence of pancreatic cancer has increased steadily over the past four decades in many parts of the world. It became the sixth or even the fifth cause of death from cancer in Western countries, according to the latest reports [2].

Even more, in 2000 worldwide there were 216.400 new diagnosed cases and 215.500 deaths from this disease, while in the United States it was estimated that in 2004 there were 31.860 new cases and 31.270 deaths, suggesting that in fact incidence and mortality are similar for this disorder [3].

Further more, the fatality rates in this disease are correlating with age, varying from 3.1 and 20.8 per 100.000 people, with a corresponding peak for the patients between 60 and 80 years old [4,5].

A serious disadvantage in the treatment of the pancreatic cancer is the fact that the diagnosis is established too late, despite the development of new technologies [6,7]. In this way, it is know that the main symptoms (e.g. pain and jaundice) appear later in the evolution, when the tumor is already locally advanced and unresectable, which makes the prognosis of the disorder quite grim [8]. It is also estimated that almost $95 \%$ of the patients are presenting to the doctor in the advanced and unresectable stages of the disease $[9,10]$.

In addition, as the current diagnostic methods and techniques can not accurately assess all the pancreatic tumors and also taking into consideration that the evolution and improvement of various surgical techniques may have reached a maximum, all hopes are now turning to an early diagnosis or in the discovery of an effective therapeutic agent. However, one way to establish a diagnosis in 
the early stage, when the cancer is potentially curable, could also be a rigorous anamnesis that would reveal the early symptoms and direct the patient to the doctor.

In the present study we used the TNM cancer staging system [11,12], which is described in details in the Methods section. In this way, the AJCC (American Joint Committee on Cancer) has developed a staging criterion for adenocarcinoma of the pancreas which follows the tumor/node/metastasis (TNM) system [13]. Although the TNM staging criteria for pancreatic cancer in the 7th edition of the AJCC Cancer Staging Manual have taken into account the fact that tumors of the pancreas are evaluated preoperatively by CT or MRI in order to determine the resectability status, these staging criteria also include information that can be determined only through postsurgical pathological evaluation of resected tumor. In addition, recent validation of concordance between AJCC stage and OS has been provided through the evaluation of 121713 patients with pancreatic adenocarcinoma included in the National Cancer Data Base (NCDB) [14].

\section{Methodology}

This analysis was carried between January 2000 to December 2005, on a sample consisting of 188 patients from Clinical Emergency Hospital "Sf. Spiridon, Iasi”, Romania, all with solid form of pancreatic cancer. 97 of the patients were females, 91 males. All the patients included in this group were diagnosed with solid pancreatic tumors, all malignant (largely represented pancreatic adenocarcinoma), which in practice predispose to confusion and even to impossibility to diagnosis, even at the time of the surgery. Cystic forms (pancreatic pseudocyst, pancreatic cystadenocarcinoma) and benign forms (chronic pancreatitis - pseudotumoral form) were excluded.

Regarding the TNM classification for pancreatic cancer [11,12] and the anatomic stage/prognostic groups, these can be found in the Tables below:

\section{Table 1. TNM Classification for Pancreatic Cancer}

\section{Primary tumor $(\mathrm{T})$}

TX Primary tumor cannot be assessed

T0 No evidence of primary tumor

Tis Carcinoma in situ

T1 Tumor limited to the pancreas, $\leq 2 \mathrm{~cm}$ in greatest dimension

T2 Tumor limited to the pancreas, $>2 \mathrm{~cm}$ in greatest dimension

T3 Tumor extends beyond the pancreas but without involvement of the celiac axis or the superior mesenteric artery

T4 Tumor involves the celiac axis or the superior mesenteric artery (unresectable primary tumor)

\section{Regional lymph nodes (N)}

NX Regional lymph nodes cannot be assessed

N0 No regional lymph node metastasis

N1 Regional lymph node metastasis

\section{Distant metastasis (M)}

M0 No distant metastasis

M1 Distant metastasis 
Table 2. Anatomic stage/prognostic groups

$\begin{array}{llll}\text { Stage } & \text { T } & \text { N } & \text { M } \\ \text { 0 } & \text { Tis } & \text { N0 } & \text { M0 } \\ \text { IA } & \text { T1 } & \text { N0 } & \text { M0 } \\ \text { IB } & \text { T2 } & \text { N0 } & \text { M0 } \\ \text { IIA } & \text { T3 } & \text { N0 } & \text { M0 } \\ & \text { T1 } & \text { N1 } & \text { M0 } \\ \text { IIB } & \text { T2 } & \text { N1 } & \text { M0 } \\ & \text { T3 } & \text { N1 } & \text { M0 } \\ \text { III } & \text { T4 } & \text { Any N } & \text { M0 } \\ \text { IV } & \text { Any T } & \text { Any N } & \text { M1 }\end{array}$

\section{Results}

In this way, the structure of the two research groups was quite identical when it comes to the sex ratio (51.6 \% females vs. $48.4 \%$ males), suggesting that pancreatic neoplasm occurs more or less equally in males and females, at least in our research groups.

Also, regarding the age of our research groups used in the present study, the median age was 65 years old. Also, the mean for the Weibull distribution was 69 years old.

In addition, the distribution of the patients in our groups, as judged by the area of origin did not revealed differences between patients originating from urban areas vs. patients from rural areas ( $53.2 \%$ from rural environment vs. $46.8 \%$ from urban environment).

Also, regarding the distribution by tumor location in the pancreas, $74.6 \%$ of the patients in our study had the tumor located in the head of the pancreas. Also, only $9.7 \%$ of the tumors are located in the body of the pancreas and only $2.2 \%$ of the patients are suffering from pancreatic cancer located in the tail of the pancreas.

Moreover, when we determined the ddistribution by the existence of the metastases at the time of the diagnosis, we observed that $62.1 \%$ of the existing tumors are located in the liver and $21.2 \%$.are located into the peritoneum.

In addition, when we looked for the distribution by size and extension of the tumor ( $\mathrm{T}$ from TNM classification), our data showed that T4 tumor staging predominates (63.8\%) (Figure 1). This could suggest that the primary tumor has a large size, being deeply grown into the organ, and into the nearby tissues. In this way, the higher the $\mathrm{T}$ number, the larger the tumor and/or the more it has grown into nearby tissues.

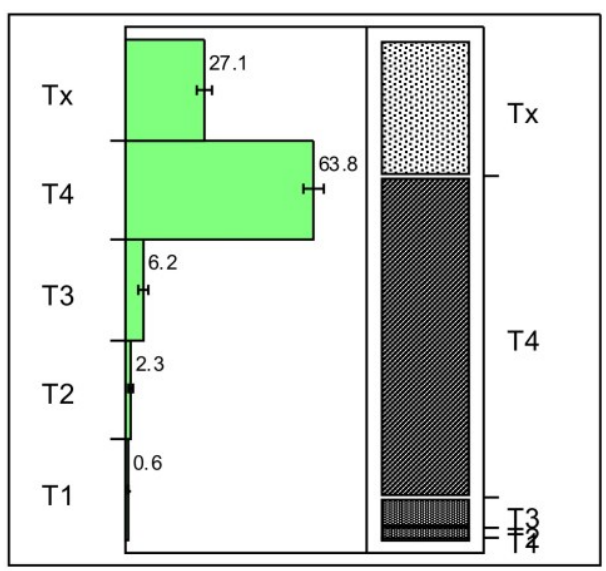

Figure 1. Distribution by the dimension and extension of the tumor (T from TNM classification) (tumor-T) for the selected patients. 
The $\mathrm{N}$ category describes whether the cancer has spread into nearby lymph nodes. Thus, regarding $\mathrm{N}$ in TNM classification, in an overwhelming proportion the $\mathrm{Nx}$ stage is predominant $(88.6 \%)$, which could suggest that regional lymph nodes cannot be assessed. Moreover, in only $10.8 \%$ of the patients no regional lymph node metastasis was found (Figure 2).

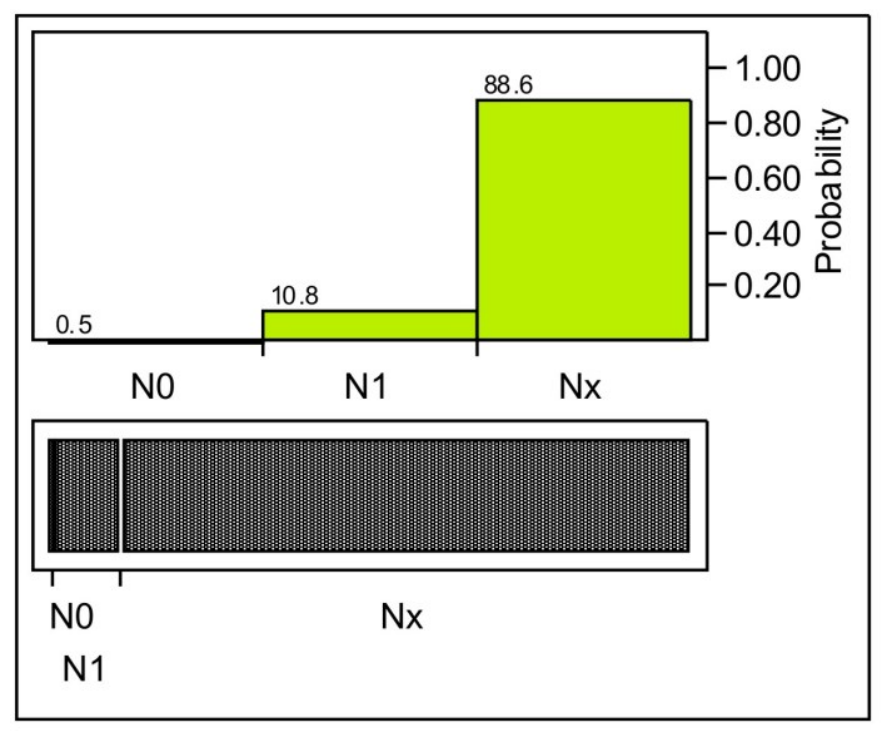

Figure 2. Distribution of the adenopathies $(\mathrm{N})$ for the selected patients.

Finally, regarding the $\mathrm{M}$ stage from the TNM cancer staging, we observed that in $62.7 \%$ of the patients no distant cancer spread was found (M0). In addition, in $37.3 \%$ it was observed that the cancer has spread to distant organs or tissues (e.g. distant metastases were found).

\section{Discussion}

Probably the biggest unresolved dilemma in the management of patients diagnosed with pancreatic tumors is the establishing of a positive diagnostic, a fact which is often impossible even intra-operatively. The differentiation between chronic pancreatitis and pancreatic neoplasm is also a big problem and there are currently cases where only the test of time (e.g. time evolution) establishes the diagnosis.

In this way, we found no significant differences between the males and females regarding the appearance of the pancreatic cancer in our sample, which could suggest that pancreatic neoplasm occurs about equally in both sexes. Still, other studies found that men are more predisposed to pancreatic cancer in some countries, as compared to women [15]. This claim could be based on some of the main risk factor for pancreatic cancer, such as the tobacco smoking, which could explain some of the aforementioned international variations and gender differences [16].

Also, in our sample the median age was 65 years old. Other studies showed that fewer than $10 \%$ of cases of pancreatic cancer occur among individuals younger than 55 years old, and the median age of onset is 71 years. In addition, the risk of pancreatic cancer increases with age, and it reaches its maximum between 70 and 75 years old [17].

We showed also no differences between patients with rural or urban origin, in regards to the pancreatic cancer diagnostic. In our sample $53 \%$ of the patients had rural origin and $47 \%$ urban origin. In fact, this findings come in contrast to other studies from other countries, which showed that the rate of pancreatic cancer in urban areas was about 2-4 fold higher than that in rural areas [18].

Regarding the pancreatic tumor location, by far the most frequent was the cephalic location (75\%). Moreover, these finding aligns with the data in the literature, since for example by using electronic information from the United States National Cancer Database, Sener et al. presented in June 1999 a report on the treatment and survival in a group of 100313 patients diagnosed with 
pancreatic cancer between 1985-1995, in which the tumour localization was $78 \%$ cephalic, $11 \%$ corporeal and $11 \%$ caudal [19].

Moreover, when study the distribution by presence of metastases at the time of diagnosis, we observed that $62.1 \%$ are located in the liver and $21.2 \%$ into the peritoneum. Unfortunately, at the time of diagnosis, patients present in advanced, unresectable stages. Even more, the Romanian hospital treats patients based on the addressability to the doctor with no prior selection of cases compared to other countries, where patients are diagnosed in different hospitals and only the potentially resectable ones are brought to a pancreatic surgery specialized center.

In fact, this is reflected very well in the distribution by the types of operations performed at the hospitals. Thus, in our sample we observed an increased number of exploratory laparotomy (20.9\%) and palliative surgery (biliary-digestive anastomosis $48.1 \%$ ), while the predominant group in other countries is the cephalic pancreaticoduodenectomy (e.g. Whipple - 66.4\%) [20].

It should be mentioned that the American Joint Commission on Cancer (AJCC) tumour node metastasis (TNM) staging system is the "language of cancer" by which physicians communicate about the disease process [21]. In this way, staging ensures an objective, reproducible classification of the extent of disease and is our most important determinant of treatment recommendations. Moreover, for research purposes, clinical trials depend on staging for appropriate patient stratification upon which accurate measurement of the treatment effect under study can be performed. Also, perhaps most importantly to the patient, staging is the best tool to provide prognostic information in our effort to manage patient expectations, especially in the context of a suggested treatment plan [22].

In this way, the ductal adenocarcinoma of the pancreas was staged according to the TNM (tumour grade, nodal status and metastases) system, where T1-T3 constitutes a resectable tumour. Moreover, T4 indicates a tumour that has invaded either the superior mesenteric artery or the celiac artery and such tumors are considered unresectable. Also, T1-T3 (with or without nodal involvement) comprise stages IA-IIB in the TNM staging system [23].

As mentioned, in our research group T4 stage tumor predominated (63.8\%). Unfortunately, this aspect suggested that the tumour is already unresectable at this stage. The next tumour stage is Tx $(27.1 \%)$, where the primary tumour cannot be assessed. Moreover, in only $6.2 \%$ of the patients the tumour extends beyond the pancreas, but without involvement of the celiac axis or the superior mesenteric artery.

In regards to the regional lymph nodes, $\mathrm{N}$ in $\mathrm{TNM}$ classification, we observed that in an overwhelming proportion $\mathrm{Nx}$ stage predominates $(88.6 \%)$, which could suggest that the regional lymph nodes cannot be assessed. Moreover, in only $10 \%$ of the patients no regional lymph node metastasis was found. In this way, the confusion and even the impossibility to diagnose even at the time of the surgery the exact extent of the tumor or of the regional lymph nodes make the prognosis for pancreatic cancer so grim.

In addition, no distant metastasis was found in $63 \%$ of patients, by using $M$ in TNM classification. Only in $37 \%$ distant metastasis was found. Thus, the presence or the absence of distant metastases was found to be a good prognosis factor for the overall survival [24].

\section{Conclusions}

Our results are showing the specific features of the distribution of the Romanian patients which suffered from pancreatic cancer. Thus, in our sample the median age was 65 years old. In addition, $53 \%$ of the patients had rural origin and $47 \%$ urban origin. Also, regarding the pancreatic tumor location, by far the most frequent was the cephalic location (75\%). Moreover, when we study the distribution by presence of metastases at the time of diagnosis, we observed that $62.1 \%$ are located in the liver and $21.2 \%$ into the peritoneum. In addition, when we staged the ductal adenocarcinoma of the pancreas according to the TNM (tumour grade, nodal status and metastases) system, where T1-T3 constitutes a resectable tumour and T4 indicates an unresectable tumour, we found that group 
T4 stage tumor predominated (63.8\%), while in regards to the regional lymph nodes (e.g. $\mathrm{N}$ in TNM classification) we observed that in an overwhelming proportion $\mathrm{Nx}$ stage predominates (88.6\%). Moreover, no distant metastasis was found in $63 \%$ of patients, by using M in TNM classification.

\section{References}

[1]. H. Beger, Treatment of pancreatic cancer: challenge of the facts, World J Surg. 27 (2003) 10751084.

[2]. V. Surlin et al., Prognostic factors in resectable pancreatic cancer, Rev. Med. Chir. Soc. Med. Nat. Iasi. 118 (2014) 924-931.

[3]. R. Pezzilli, Screening tests for pancreatic cancer: searching for the early symptoms or the population at risk, Jop. 5 (2004) 240-242.

[4]. E. Tamm, Diagnosis, staging, and surveillance of pancreatic cancer, AJR Am J Roentgenol. 180 (2003) 1311-1323

[5]. D. Timofte et al., Molecular factors with predictive value for the survival rate in pancreatic cancer: focusing on CA 19-9, Analele Ştiinţifice ale Universităţii „Alexandru Ioan Cuza”, Secţiunea Genetică şi Biologie Moleculară. 15 (2014) 21-26.

[6]. D. Timofte et al., The relevance of some tumoral markers in patients with pancreatic cancer, Analele Ştiinţifice ale Universităţii „Alexandru Ioan Cuza”, Secţiunea Genetică şi Biologie Moleculară, 15 (2014) 51-58.

[7]. D. Timofte et al., The relevance of some molecular markers in recurrent pancreatic cancer: focusing on CA 19-9 and cytokeratins, Rev. Med. Chir. Soc. Med. Nat. Iasi. 119 (2015) 730737.

[8]. D. Timofte et al., Studying the Post-Operatory and Molecular Modifications in the Chronic Pancreatitis and Pancreatic Cancer - The Importance of the Micronutrients and Pancreatic Enzyme Supplementation, International Letters of Natural Sciences. 47 (2015) 89-96.

[9]. D. Timofte, L. Ionescu, L. Ochiuz, Mini-Review on the Glucose Metabolism Modifications after Pancreatic Resection, International Letters of Natural Sciences. 53 (2016) 65-71.

[11]. National Comprehensive Cancer Network, NCCN Clinical Practice Guidelines in Oncology: Pancreatic Adenocarcinoma, 2015.

[12]. American Joint Committee on Cancer (AJCC) TNM staging system. American Cancer Society, 2013.

[13]. S. Edge, D. Byrd, C. Compton CC, AJCC Cancer Staging Manual (ed 7th). New York: Springer; (2010).

[14]. K. Bilimoria, D. Bentrem, C. Ko CY, Validation of the 6th edition AJCC Pancreatic Cancer Staging System: report from the National Cancer Database, Cancer. 110 (2007) 738-744.

[15]. D. Michaud, Epidemiology of pancreatic cancer, Minerva Chir. 59 (2004) 99-111.

[16]. M. Edderkaoui, E. Thrower, Smoking induced pancreatitis and pancreatic cancer, Pancreapedia: Exocrine Pancreas Knowledge Base, 2015.

[17]. D. Yadav, A. Lowenfels, The Epidemiology of Pancreatitis and Pancreatic Cancer. Gastroenterology. 144 (2013) 1252-1261.

[18]. L. Wang, G. Yang, X. Lu, Z. Huang, H. Li, Pancreatic cancer mortality in China, World J Gastroenterol. 9 (2003) 1819-1823. 
[19]. S. Sener, Pancreatic cancer: a report of treatment and survival trends for 100,313 patients diagnosed from 1985-1995, using the National Cancer Database, J. Am Coll. Surg. 189 (1999) $1-7$.

[20]. M. Del Chiaro, S. Presciuttini, L. Bertacca, A. Zerbi A, Clinical Pancreatic Cancer. HPB (Oxford). 7 (2005) 76-90.

[21]. F. Greene, L. Sobin, The staging of cancer: a retrospective and prospective appraisal, CA Cancer J Clin.58 (2008) 180-190.

[22]. W. Nabil, Impact of Tumor Grade on Prognosis in Pancreatic Cancer: Should We Include Grade in AJCC Staging? Annals of Surgical Oncology. 17 (2010): 2312-2320.

[23]. S. Chakraborty, S. Singh, Surgical resection improves survival in pancreatic cancer patients without vascular invasion- a population based study, Ann Gastroenterol. 26 (2013) 346-352.

[24]. S. Yamada, T. Fujii, H. Sugimoto H, Pancreatic cancer with distant metastases: a contraindication for radical surgery? Hepatogastroenterology. 56 (2009) 881-885. 